\title{
Dynamical ejecta from precessing neutron star-black hole mergers with a hot, nuclear-theory based equation of state
}

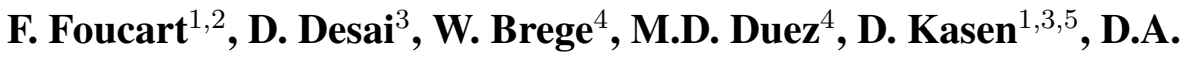 \\ Hemberger $^{6}$, L. E. Kidder ${ }^{7}$, H.P. Pfeiffer ${ }^{8}$, M.A. Scheel ${ }^{6}$ \\ ${ }^{1}$ Nuclear Science Division, Lawrence Berkeley National Laboratory, 1 Cyclotron Rd, \\ Berkeley, CA 94720, USA \\ ${ }^{2}$ NASA Einstein Fellow \\ ${ }^{3}$ Department of Physics, University of California, Berkeley, Le Conte Hall, Berkeley, CA \\ 94720 \\ ${ }^{4}$ Department of Physics \& Astronomy, Washington State University, Pullman, Washington \\ 99164, USA \\ ${ }^{5}$ Astronomy Department and Theoretical Astrophysics Center, University of California, \\ Berkeley, 601 Campbell Hall, Berkeley CA, 94720 \\ ${ }^{6}$ TAPIR, Walter Burke Institute for Theoretical Physics, MC 350-17, California Institute of \\ Technology, Pasadena, California 91125, USA \\ ${ }^{7}$ Center for Radiophysics and Space Research, Cornell University, Ithaca, New York, 14853, \\ USA \\ ${ }^{8}$ Canadian Institute for Theoretical Astrophysics, University of Toronto, Toronto, Ontario \\ M5S 3H8, Canada
}

\begin{abstract}
Neutron star-black hole binaries are among the strongest sources of gravitational waves detectable by current observatories. They can also power bright electromagnetic signals (gamma-ray bursts, kilonovae), and may be a significant source of production of r-process nuclei. A misalignment of the black hole spin with respect to the orbital angular momentum leads to precession of that spin and of the orbital plane, and has a significant effect on the properties of the post-merger remnant and of the material ejected by the merger. We present a first set of simulations of precessing neutron star-black hole mergers using a hot, composition dependent, nuclear-theory based equation of state (DD2). We show that the mass of the remnant and of the dynamical ejecta are broadly consistent with the result of simulations using simpler equations of state, while differences arise when considering the dynamics of the merger and the velocity of the ejecta. We show that the latter can easily be understood from assumptions about the composition of low-density, cold material in the different equations of state, and propose an updated estimate for the ejecta velocity which takes those effects into account. We also present an updated mesh-refinement algorithm which allows us to improve the numerical resolution used to evolve neutron star-black hole mergers.
\end{abstract}

PACS numbers: 04.25.dg, 04.40.Dg, 26.30.Hj, 98.70.-f 


\section{Introduction}

The merger of a neutron star-black hole (NSBH) binary is one of the strongest source of gravitational waves detectable by the current generation of ground-based observatories (Advanced LIGO [1], Advanced Virgo [2], KAGRA [3]). The recent detection by advanced LIGO of two merging black hole binaries opened the era of gravitational wave astronomy [4, 5]. With a growing number of detectors and the improved sensitivities expected in the coming years, many more binary black holes will probably be observed [6]. Gravitational waves from mergers involving neutron stars (binary neutron stars and/or NSBH binaries) are also expected in the near future [7].

In the presence of at least one neutron star, information complementing the gravitational wave signal can be obtained from powerful electromagnetic signals which are expected to follow the mergers. Short gamma-ray bursts are generally thought to be powered by neutron star mergers [8, 9, 10, 11], while the ejection of neutron rich material by some mergers can produce bright optical/infrared transients on a timescale of days after the merger (a kilonova, or macronova) [12, 13, 14, 15, 16]. These electromagnetic signals could provide information about the localization and environment of the merger, as well as the parameters of the merging objects. Additionally, r-process nucleosynthesis in the ejected material results in the production of many heavy elements whose origin remains uncertain today [17, 18]. Determining whether neutron star mergers are the main site of production of r-process elements is an important open problem in nuclear astrophysics today. The observation of neutron star mergers can also help us constrain the equation of state of matter in the cold, dense, neutron rich core of neutron stars [19, 20, 21, 22, 23], and thus probe the strength of nuclear forces in conditions otherwise inaccessible to us.

General relativistic simulations of compact binary mergers are critical to understand the dynamics of mergers, and the properties of the post-merger remnant and ejected material. They are also needed to obtain reliable templates for the gravitational wave signals emitted by mergers. Numerical simulations so far have shown that for circular binaries, a NSBH merger can result either in the rapid disruption of the neutron star by the black hole, or a direct plunge of the neutron star into the black hole as the neutron star reaches the innermost stable circular orbit (ISCO) of the black hole. The competition between the binary separation at tidal disruption and the location of the ISCO is thus the main determinant of the outcome of the merger. Practically, the most important parameters in the determination of the merger outcome are the radius of the neutron star (larger stars favor disruption), the mass of the black hole (more massive black holes hinder disruption), and the spin of the black hole (a larger aligned component of the black hole spin helps disruption) [24, 25].

If the neutron star is tidally disrupted, some material is immediately unbound by the merger (dynamical ejecta) [26, 27], some material is placed on highly eccentric bound orbits (bound tail), some forms a hot accretion disk around the black hole, and most of the matter is accreted by the black hole within $\sim 1 \mathrm{~ms}$. Long term evolutions of remnant accretion disks show that magnetically-driven winds [28], neutrino-driven winds [29, 30], and energy deposition due to magnetic turbulence and nuclear recombination [31, 30] can combine to 
unbind a significant fraction of the remnant disk over timescales of up to a few seconds (disk outflows, for $\sim 5-20 \%$ of the disk mass at least). One of the main objective of merger simulations is to determine the mass and properties (velocity, composition, entropy) of the dynamical ejecta and disk outflows, as these have a significant impact on the brightness, color, and duration of kilonovae [32, 33], and on the abundance of the various elements produced through r-process nucleosynthesis [18, 34].

Our current ability to accurately predict the result of a NSBH merger is largely limited by two factors: the complexity of the physical processes which should be included in numerical simulations, and the wide parameter space that we need to explore. The most important parameters to vary are the masses of the compact objects, the spin magnitude and orientation of the black hole, and the unknown equation of state of the neutron star [25]. Neutron star spins and the orbital eccentricity of the binary [35] could also play an important role, especially for binaries formed through dynamical interactions in dense clusters.

The impact of the orientation of the black hole spin has only been studied by general relativistic simulations using either an ideal gas equation of state [36, 26], or piecewise polytropic equations of state fitted to nuclear theory for cold matter in beta-equilibrium, but without any composition information and with a very idealized temperature dependence of the equation of state [37]. While this provides us with good first estimates of the result of the merger, important properties of the post-merger remnant are either inaccurate (e.g. velocity and geometry of the dynamical ejecta), or inaccessible (e.g. composition, neutrino cooling) without the use of a more advanced description of the neutron star matter. In general, at fixed spin magnitude, the main consequence of a misaligned black hole spin is to make the disruption of the neutron star less likely. This has been observed both in fully general relativistic simulations [36, 26, 37], as well as in smoothed particle hydrodynamics simulations of higher mass ratio binaries performed in a fixed Kerr metric [38].

Here, we study the properties of the dynamical ejecta and the formation of an accretion disk in a small number of NSBH mergers with misaligned spins using the hot, composition dependent $D D 2$ equation of state [39]. We can then compare our results with the larger database of precessing NSBH simulations recently published by Kawaguchi et al. [40], as well as the semi-analytical model for the properties of the ejected matter derived from these simulations [41].

The post-merger evolution of NSBH binaries is significantly affected by both magnetic fields and neutrinos. In recent years, numerical simulations have rapidly improved their treatment of these complex physical effects in NSBH mergers. Magnetic fields can power magnetically dominated outflows ("proto-jets") [42], while neutrinos play an important role in the cooling of the post-merger disk and the evolution of the composition of the fluid [43]. Both magnetic fields [28] and neutrino absorption [29, 30, 43] can drive disk winds after the merger. The dynamics of the merger and the properties of the dynamical ejecta are, on the other hand, largely unaffected by magnetic fields. The dynamical ejecta is also generally too cold, and moving away from the remnant too fast, to undergo significant changes of composition due to neutrino-matter interactions.

For this study of the merger dynamics and tidal disruption of NSBH binaries, we thus 
Table 1. Initial parameters of the binaries studied in this paper. $M_{\mathrm{BH}}$ is the Christodoulou mass of the black hole, $M_{\mathrm{NS}}$ the ADM mass of an isolated neutron star with the same equation of state and baryon mass as the neutron star under consideration, $\chi_{\mathrm{BH}}$ is the dimensionless spin of the black hole, $i_{\mathrm{BH}}$ is the initial inclination between the black hole spin and the orbital angular momentum, $N_{\text {orbits }}$ is the number of orbits up to the point at which $0.01 M_{\odot}$ has been accreted by the black hole, $\Omega_{0}$ is the initial angular velocity, and $M=M_{\mathrm{BH}}+M_{\mathrm{NS}} . \Delta x_{\mathrm{dis}}$ is the typical grid resolution in the laboratory frame for the finest level of refinement used during the disruption of the neutron star (see Appendix A for more detail on the grid structure).

\begin{tabular}{c|ccccccc} 
Model & $M_{\mathrm{BH}}\left(M_{\odot}\right)$ & $M_{\mathrm{NS}}\left(M_{\odot}\right)$ & $\chi_{\mathrm{BH}}$ & $i_{\mathrm{BH}}(\mathrm{deg})$ & $N_{\text {orbits }}$ & $\Omega_{0} M$ & $\Delta x_{\text {dis }}(\mathrm{m})$ \\
\hline M5-S7-I60 & 5 & 1.4 & 0.7 & 60 & 3.3 & 0.0416 & 170 \\
M5-S9-I60 & 5 & 1.4 & 0.9 & 60 & 3.7 & 0.0415 & 160 \\
M7-S7-I60 & 7 & 1.4 & 0.7 & 60 & 3.1 & 0.0479 & 190 \\
M7-S9-I60 & 7 & 1.4 & 0.9 & 60 & 4.2 & 0.0478 & 170 \\
M7-S9-I20 & 7 & 1.4 & 0.9 & 20 & 4.8 & 0.0474 & 160
\end{tabular}

choose to neglect the effects of magnetic fields. Additionally, we only include neutrino cooling through the use of a simple leakage scheme [44, 45, 46], in which the energy and lepton number carried away by neutrinos is estimated from the local properties of the matter and an estimate of the optical depth of the fluid. While more advanced neutrino transport schemes have recently been implemented in merger simulations [43, 47, 48, 49], neutrino-matter interactions will not play any significant role in a NSBH merger before the formation of a hot accretion disk. We only include the neutrino leakage scheme for its ability to approximately capture neutrino cooling in the post-merger remnant, and because the computational cost of the leakage scheme is small. Details of our implementation of the leakage scheme can be found in previous publications [50, 51].

We first provide a description of our initial conditions in Sec. 2. The physical results (merger dynamics, ejecta properties, post-merger evolution) are discussed in Sec. 3, while Sec. 4 provides a more in-depth comparison with previous results. A brief summary and concluding remarks are provided in Sec. 5. We also provide in the Appendix a description of an improved method to adaptively modify our computational grid, and a discussion of the expected numerical accuracy of our simulations.

\section{Initial Conditions}

We consider a series of NSBH binaries in which we modify the black hole mass and spin. We fix the ADM mass of the neutron star (at infinite separation) to $M_{\mathrm{NS}}=1.4 M_{\odot}$, and use the DD2 equation of state [39]. This leads to neutron stars of areal radius $R_{\mathrm{NS}}=13.2 \mathrm{~km}$, at the high end of the range of values compatible with nuclear theory and astrophysical constraints [52]. The Christodoulou mass of the black hole is either $M_{\mathrm{BH}}=5 M_{\odot}$ or $M_{\mathrm{BH}}=$ $7 M_{\odot}$, covering the lower range of black hole masses observed in X-ray binaries [53, 54]. Higher mass black holes do not lead to the disruption of the neutron star, except for near- 
extremal, aligned black hole spins. The dimensionless spin of the black hole is either $\chi_{\mathrm{BH}}=0.7$ or $\chi_{\mathrm{BH}}=0.9$. The spin of black holes in NSBH binaries is entirely unknown, and the choice of moderate to high spins is made in order to cover the expected range of spins over which the neutron star disrupts. Finally, the inclination of the spin with respect to the initial angular momentum of the system, in the coordinates of our initial data, is set to $i_{\mathrm{BH}}=60^{\circ}$, except for a high-mass, high-spin case in which we set $i_{\mathrm{BH}}=20^{\circ}$. A summary of the simulations considered here is provided in Table 1 . In the rest of the text, we label each configuration by the mass of the black hole, the spin of the black hole, and the inclination of that spin, e.g. M5-S7-I60 corresponds to a simulation with $M_{\mathrm{BH}}=5 M_{\odot}, \chi_{\mathrm{BH}}=0.7$, and $i_{\mathrm{BH}}=60^{\circ}$.

The initial data is generated using our in-house elliptic solver [55, 56], which includes an iterative algorithm to solve for the constraints in Einstein's equations, while requiring that the binary components have the requested masses and spins, that the binary is in a quasicircular orbit (effectively leading to small eccentricities $e \sim 0.01$ ), and that the fluid is in hydrostatic equilibrium and the flow of the neutron star matter is irrotational [57, 58]. We consider relatively close initial separations, as we are more interested in the impact of a hot, nuclear theory based equation of state on the dynamics of the merger than in the production of long, accurate waveforms. The systems considered here evolve for $3-5$ orbits before the neutron star is disrupted by the tidal field of the black hole (or plunges into the black hole).

\section{Results}

We evolve the coupled system of Einstein's equation of general relativity and the general relativistic equations of hydrodynamics using the SpEC code [59]. SpEC uses pseudospectral methods to evolve Einstein's equations in the Generalized Harmonics formalism [60, 61], and finite volume methods to evolve the fluid using high-order shock capturing methods. The two systems of equations are coupled through interpolation of the metric and fluid variables at the end of each time step. The magnitude and orientation of the spin in the simulations are measured by using approximate rotational Killing vectors [62]. A detailed description of our numerical methods can be found in previous publications [63, 26].

\subsection{Late inspiral and merger dynamics}

The orbital evolution of the binary before merger proceeds as expected, with significant precession of the spin orientation and of the orbital plane even for the relatively short simulations considered here. Fig. 1 shows the evolution of the inclination of the black hole spin with respect to the initial direction of the total angular momentum of the system, and the phase of the precession of the black hole spin. The first is nearly constant, while the second shows that for the longest simulation studied here (M7-S9-I20), we observe about three-quarters of a precession period. The orientation of the orbital plane, which we do not show here, precesses at the same frequency as the black hole spin. These effects can be entirely understood from post-Newtonian predictions for the evolution of point particles, as 

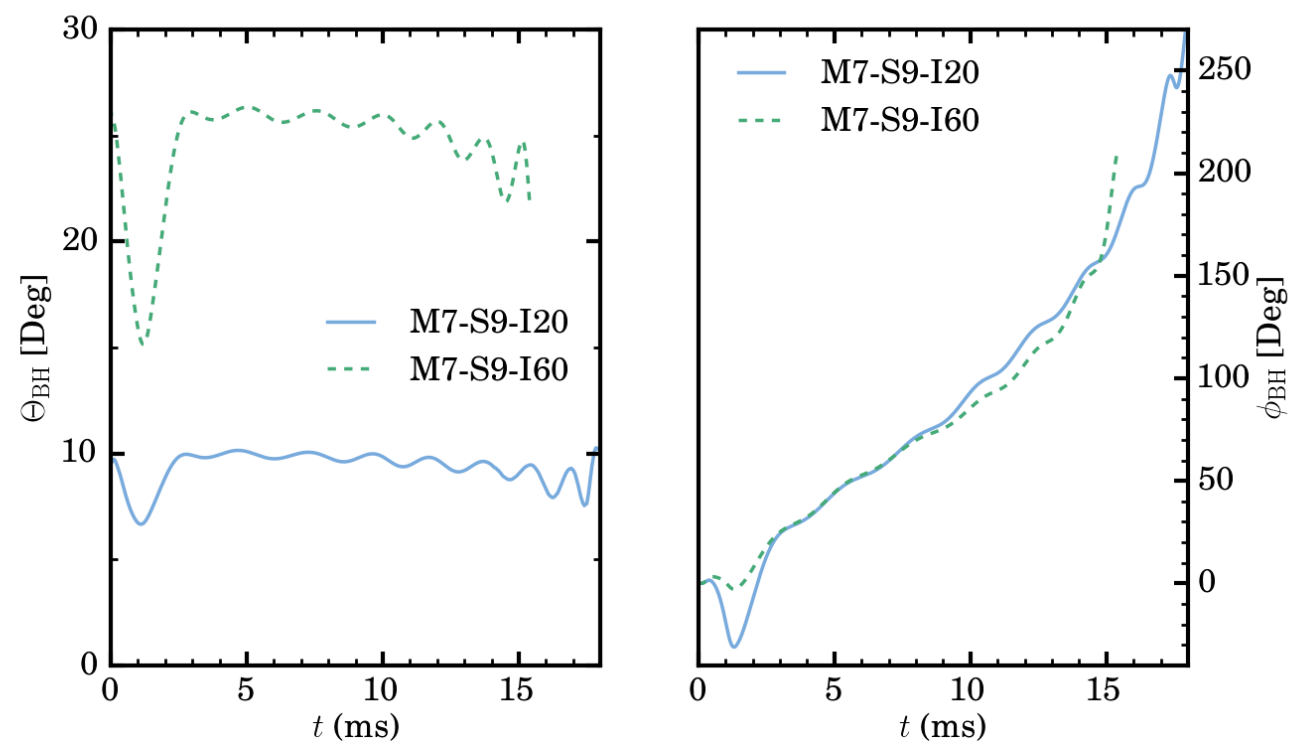

Figure 1. Inclination angle $\Theta_{\mathrm{BH}}$ of the black hole spin with respect to the initial direction of the total angular momentum of the system (left), and phase $\phi_{\mathrm{BH}}$ of the precession of the black hole spin around the total angular momentum (right). As expected, $\Theta_{\mathrm{BH}}$ is nearly constant in time (up to the impact of the loss of angular momentum due to gravitational wave emission). We show results for the two longest simulations, M7-S9-I60 and M7-S9-I20. Over the course of the evolution, the binary goes through $0.5-0.75$ spin precession periods. The rapid variation of the measured inclination at early times is due to a smooth change in the gauge condition between the initial data and the evolution, and shows how coordinate-dependent these measurements can be in a truly dynamical spacetime.

already shown for simpler equations of state [36].

Among the simulations considered here, one falls within the range of parameters for which we expect direct plunge of the neutron star into the black hole (M7-S7-I60), and the others are expected to disrupt (although M7-S9-I60 is close to the limit between disrupting and plunging neutron stars) [25]. We find indeed that, at the accuracy at which we can resolve the merger, there is no significant amount of matter remaining outside of the black hole after the merger of model M7-S7-I60. At our highest resolution, the merger leaves $0.004 M_{\odot}$ of material outside the black hole (including $0.002 M_{\odot}$ of unbound material). At lower resolution, we found higher values of the remaining mass (a few percents of a solar mass). Our results are consistent with convergence of the remnant baryon mass to zero, although we cannot rule out a remnant disk and unbound ejecta at the level of $\sim 0.001 M_{\odot}$. The other systems show the expected disruption of the neutron star. The higher mass systems, however, disrupt as the neutron star plunges into the black hole, leading to a very dynamical disruption process.

Fig. 2 shows a snapshot of the baryon density for the two extreme models M7-S7-I60 and M5-S9-I60. The first leads to direct plunge of the neutron star into the black hole, while the second results in the ejection of a small amount of material $\left(M_{\mathrm{ej}} \approx 0.014 M_{\odot}\right.$, see below) 

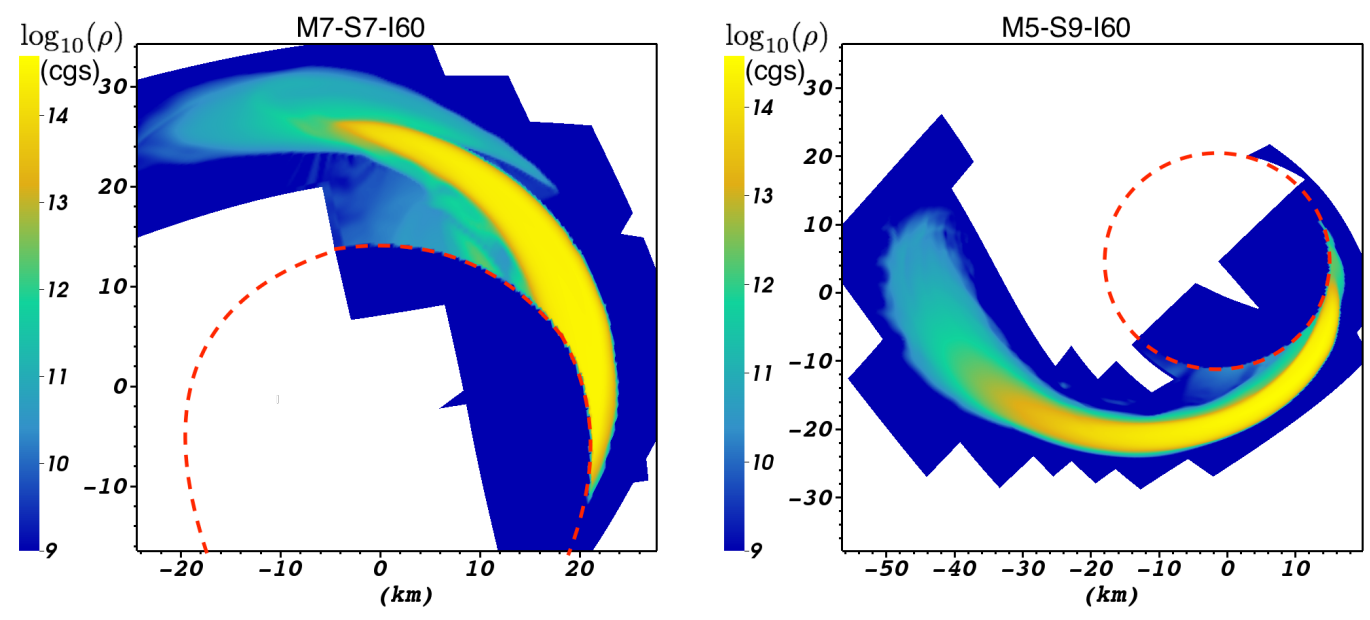

Figure 2. Barion density in the orbital plane of the merging binary at the time at which half the neutron star material has been accreted by the black hole. Left: Model M7-S7-I60. Right: Model M5-S9-I60. White regions are not covered by the finite volume grid, while the dashed red line shows the boundary of the excised region inside the black hole. Note the different length scales used for each plot. In model M7-S7-I60, the neutron star plunges fully into the black hole. In model M5-S9-I60, a very narrow tidal tail forms, with a small amount of unbound material.

and the formation of an accretion disk. The narrow tail with sharp edges which is typical of these NSBH mergers, is clearly visible for the lower mass system M5-S9-I60. We note that, while M5-S9-I60 is the most favorable system for disruption of the neutron star outside the ISCO, the disruption is still occurring very close to the black hole, and partially mixes with the plunge of the neutron star into the black hole. We can also see that, during tidal disruption, the code makes full use of the adaptive finite volume grid discussed in Appendix A (white regions are not covered by the numerical grid). The formation of a narrow tidal tail is one of the most striking difference between simulations using ideal gas or piecewise polytropic equations of state, and those using nuclear-theory, composition dependent equations of state, and was already noted in simulations of spin-aligned NSBH mergers [51]. For an ideal gas equation of state, the disruption of the neutron star is generally more gradual, and the tidal tail significantly wider, as shown in Fig.3 of Foucart et al. (2014) [51].

The main properties of the post-merger remnant and characteristics of the dynamical ejecta are summarized in Table 2, and discussed in more detail below (except for the inaccurate model M7-S9-I60, discussed in Appendix B.

\subsection{Ejecta properties}

In NSBH mergers, there are effectively two sources of ejecta. The first is the dynamical ejecta, which is produced by the tidal disruption of the neutron star, and the second is disk outflows, which are due to magnetically-driven or neutrino-driven winds in the post-merger accretion disk, and to heating of the remnant disk due to nuclear recombination. Our merger simulations 
Table 2. Properties of the dynamical ejecta and post merger remnant. $M_{\mathrm{BH}}^{f}$ and $\chi_{\mathrm{BH}}^{f}$ are the mass and dimensionless spin of the black hole, and $M_{\text {out }}^{f}$ is the baryon mass remaining outside of the black hole. Those quantities are measured at the first minima of the accretion rate onto the black hole, before circularization of the accretion disk. The baryon mass outside of the black hole immediately after disk formation (which is a more vaguely defined time) is typically $10 \%-20 \%$ lower than $M_{\mathrm{out}}^{f} . M_{\mathrm{ej}}$ is the mass of the dynamical ejecta, and $\langle v / c\rangle_{\mathrm{ej}}$, $\langle i\rangle_{\mathrm{ej}}$ are the mass-weighted average of its velocity and orbital inclination with respect to the equatorial plane of the black hole. All these properties are nearly constant, from about $1 \mathrm{~ms}$ after the merger. Bracketed numbers for $M_{\text {out }}^{f}$ and $M_{\mathrm{ej}}$ show semi-analytical predictions for the mass outside of the black hole $10 \mathrm{~ms}$ after merger [25], and the ejected mass [41], while bracketed numbers for $M_{\mathrm{BH}}^{f}$ and $\chi_{\mathrm{BH}}^{f}$ are semi-analytical predictions from [64]. The analytical values provided in this table for the final mass and spin of the black hole were kindly provided to us by Francesco Pannarale.

\begin{tabular}{c|cc|cccc} 
Model & $M_{\mathrm{BH}}^{f}\left(M_{\odot}\right)$ & $\chi_{\mathrm{BH}}^{f}$ & $M_{\mathrm{out}}^{f}\left(10^{-2} M_{\odot}\right)$ & $M_{\mathrm{ej}}\left(10^{-2} M_{\odot}\right)$ & $\langle v / c\rangle_{\mathrm{ej}}$ & $\langle i\rangle_{\mathrm{ej}}$ \\
\hline M5-S7-I60 & $6.12[6.2]$ & $0.76[0.76]$ & $15[10]$ & $1.4[0.6]$ & 0.16 & $26^{\circ}$ \\
M5-S9-I60 & $6.06[6.1]$ & $0.86[0.86]$ & $21[19]$ & $1.4[1.1]$ & 0.15 & $27^{\circ}$ \\
M7-S7-I60 & $8.22[8.3]$ & $0.75[0.75]$ & $\leq 0.5[0]$ & $\leq 0.25[0]$ & - & - \\
M7-S9-I20 & $7.75[8.0]$ & $0.90[0.93]$ & $47[31]$ & $4.3[4]$ & 0.20 & $12^{\circ}$
\end{tabular}

can only constrain the mass and properties of the dynamical ejecta. We should however keep in mind that $5-20 \%$ of the remaining accretion disk may be unbound on longer timescales, thus contributing to the total amount of ejected material [31, 30].

In our models, the mass of the dynamical ejecta vary in the range $(0.01-0.05) M_{\odot}$, with the high spin, high black hole mass systems producing the most ejecta (see Table 2). This is generally less mass than for aligned-spin systems at the same mass ratio and black hole spin [51, 37]. Indeed, tidal disruption and the ejection of material are usually favored by a higher value of the aligned component of the black hole spin.

We note that the impact of the spin on the ejected mass is large only when the neutron star disrupts close to the ISCO. Indeed, the main impact of a high spin is to bring the ISCO closer to the black hole, thus letting the neutron star disrupt deeper into the gravitational potential of the black hole. If even at low black hole spin the disruption of the neutron star occurs outside of the ISCO, the dynamics of the merger are not affected as much by the black hole spin. This is why the impact of the black hole spin on the mass of the ejecta is large for $M_{\mathrm{BH}}=7 M_{\odot}$, but small for $M_{\mathrm{BH}}=5 M_{\odot}$. In the latter case, the ejected material is unbound at a separation at which spin effects are relatively minor. Disk formation, which occurs later in the disruption of the neutron star, is significantly affected by the black hole spin for all black hole masses considered here.

As is typical for the dynamical ejecta of NSBH mergers, the unbound material is very neutron rich. The density-weighted average electron fraction of the ejecta is $\left\langle Y_{e}\right\rangle=0.04-$ 0.06 , depending on the simulation. For such neutron-rich ejecta, the outcome of r-process nucleosynthesis is guaranteed to be the robust production of heavy elements, including the 2nd and 3rd peak of the r-process, as well as high-opacity lanthanides, with little to no production 

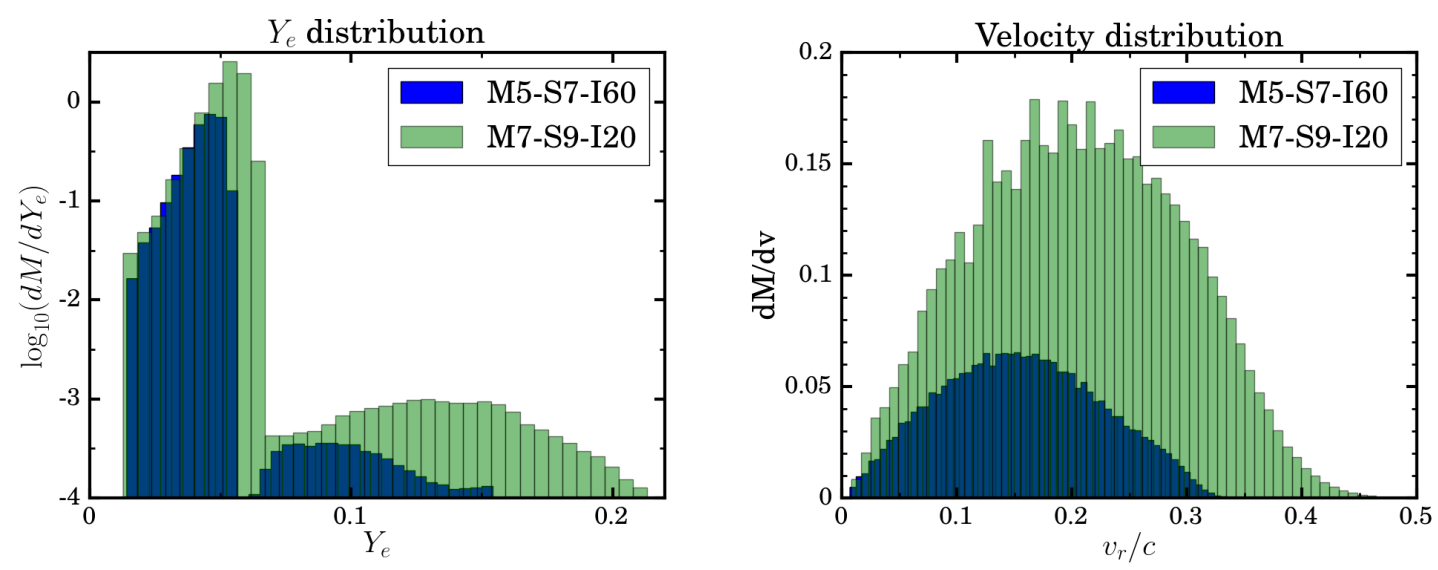

Figure 3. Distribution of the electron fraction $Y_{e}$ (Left) and asymptotic velocity (Right) in the ejecta of models M5-S9-I60 and M7-S9-I20. Most of the ejected material has $Y_{e} \leq 0.06$ and all of it has $Y_{e} \leq 0.25$. We observe a broad velocity distribution, symmetric around the average values $\langle v\rangle=(0.15,0.20)$.

of elements around the observed 1st peak of the r-process [18, 34, 65]. A more detailed description of the composition of the ejecta for two of our models is presented in Fig. 3 (left panel). We see that there is no significant amount of material with $Y_{e}>0.07$ (note the logarithmic scale used for this plot), and absolutely no observed ejecta at $Y_{e}>0.25$, where lighter r-process elements could be produced. Overall, we can thus conclude that (i) r-process nucleosynthesis in this dynamical ejecta will only produce the heavier r-process elements [34], and (ii) the opacity of the ejecta will be high, causing the electromagnetic transient powered by the r-process to peak in the infrared [32].

The asymptotic velocity of the ejecta is another important parameter if we want to predict the properties of that infrared transient. Larger velocities imply faster expansion of the ejecta, and a larger amplitude, shorter lived transient [32, 33]. The right panel of Fig. 3 shows the distribution of velocities in the ejecta of two of our models. The density-weighted average velocity of the ejecta is $\langle v\rangle \sim 0.15 c$ for $M_{\mathrm{BH}}=5 M_{\odot}$ and $\langle v\rangle \sim 0.20 c$ for $M_{\mathrm{BH}}=7 M_{\odot}$. We see that the ejecta has a broad velocity distribution, covering the range $0 \leq v \leq 2\langle v\rangle$. The measured kinetic energy of the ejecta ranges from $3 \times 10^{50}$ ergs for model M5-S9-I60 to $1.7 \times 10^{51}$ ergs for M7-S9-I20. The kinetic energy of the ejecta plays an important role as the available amount of energy to power radio transients as the ejecta interacts with the interstellar medium [66]. We note that the typical velocity of the ejecta measured here is comparable to what was obtained in spin-aligned simulations at the same mass ratio using the hot, composition dependent nuclear-theory based equation of state LS220 [51], but is lower than in simulations using a fixed fluid composition [26, 37]. We discuss the origin of this difference in Sec. 4 .

The geometry of the ejecta can also have important effects on the properties of kilonovae, mainly by introducing a dependence of the kilonova signal in the orientation of the binary with respect to the observer. In NSBH mergers, this is particularly important because the dynamical 

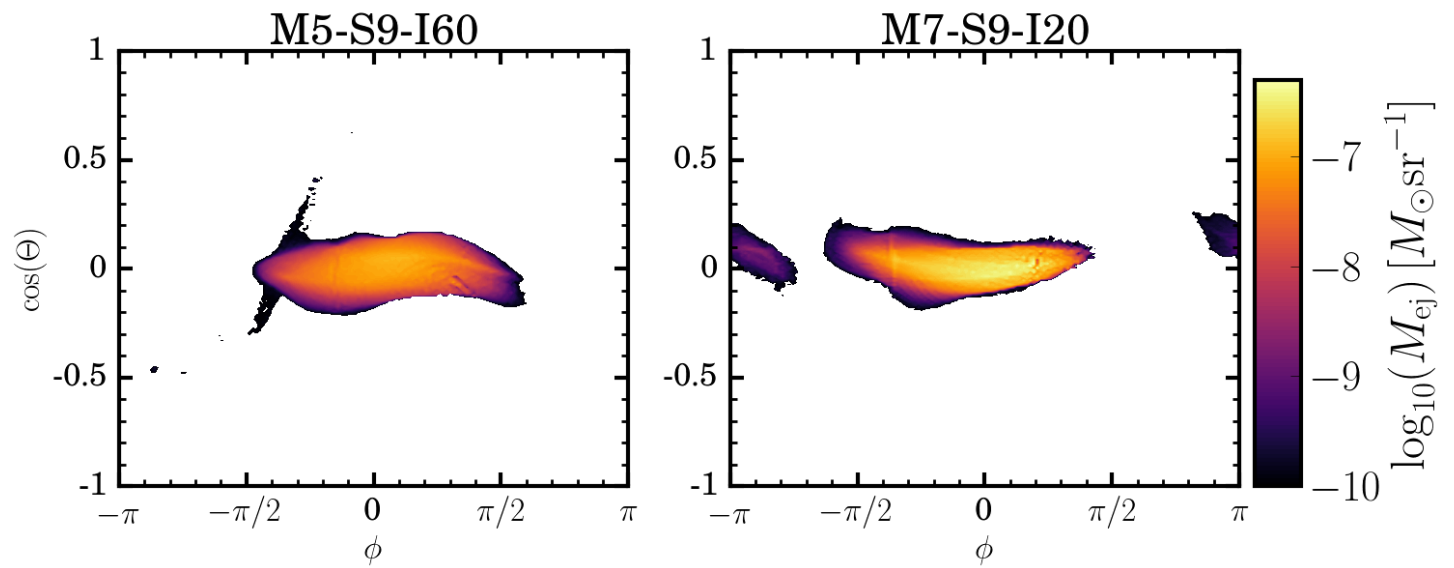

Figure 4. Angular distribution of the ejected material for simulations M5-S9-I60 (Left), and M7-S9-I20 (Right). We use spherical polar coordinates centered on the black hole, with the polar axis chosen so that the equatorial plane is the best fit plane for the orbital plane of the unbound material. The ejected material fills a narrow region of opening angle $\sim 20^{\circ}\left(\sim 180^{\circ}\right)$ in the vertical (azimuthal) direction.

ejecta is very opaque and neutron rich, while material ejected later in disk outflows could have a high enough electron fraction to prevent the production of heavy r-process elements, thus resulting in a less opaque ejecta, and a bluer, brighter, and shorter electromagnetic signal [31, 30, 67, 68, 69]. More precisely, this is more likely to happen for disk outflows in the polar regions of the post-merger remnant, and requires that the disk outflows are not masked by high-opacity dynamical ejecta.

In Fig. 4, we show the angular distribution of the dynamical ejecta, in a spherical polar coordinate system $(r, \theta, \phi)$ chosen so that the average orbital plane of the ejecta corresponds to $\theta=0$. The inclination $\langle i\rangle_{\mathrm{ej}}$ of that coordinate system with respect to the black hole spin is listed in Table 2. We see that the overall geometry of the dynamical ejecta is consistent with the results of previous NSBH merger simulations [27]. The ejecta forms a crescent with an azimuthal opening angle of $\sim 180^{\circ}$, and a vertical opening angle of $\sim 20^{\circ}$. In all of our models, the misalignment between the orbital plane of the ejecta and the equatorial plane of the spinning black hole remains small $\left(\leq 30^{\circ}\right)$, and the dynamical ejecta should thus be unable to obscure emission from polar disk outflows. While differential precession causes a slight warp of the orbital plane of the ejecta, this remains a very small effect. This is due to the fact that the ejecta rapidly leaves the strong field region in which orbital precession is significant.

We also note that there exist a potentially important correlation between the velocity of the ejecta and the azimuthal angle $\phi$, shown on Fig. 5. Not surprisingly, ejecta farthest from the bound material in the azimuthal direction is also given more kinetic energy and has a larger asymptotic velocity. The breadth of the ejected material in the radial direction will be smaller with this effect taken into account that if one assumes a single broad velocity distribution (as in e.g. [41]). The impact of this effect on kilonova lightcurves will have to be tested using 3D radiation transport. Fig. 5 indicates that a rough estimate of the range of velocity $\left(v_{\min }, v_{\max }\right)$ 

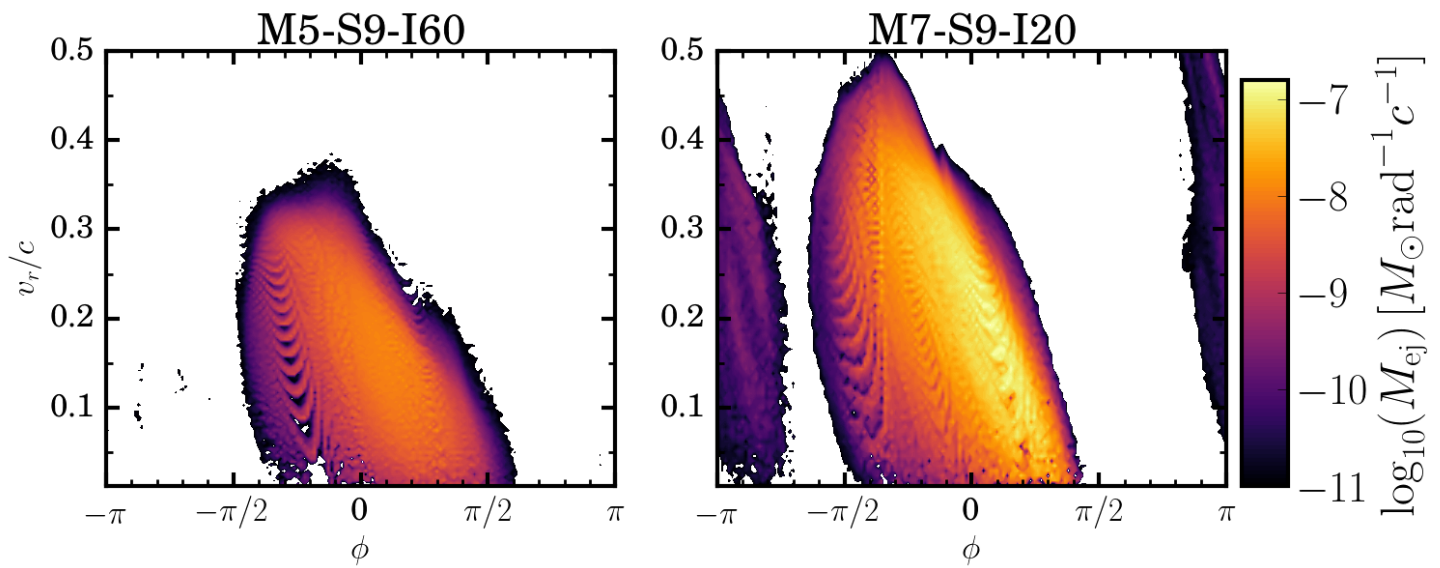

Figure 5. Distribution of the asymptotic radial velocity of the ejected material for simulations M5-S9-I60 (Left), and M7-S9-I20 (Right), as a function of the azimuthal angle $\phi$ used in Fig. 4 There is a clear linear trend in the evolution of the average velocity with azimuthal angle, which may create significant variations in the timescale of kilonovae as a function of the orientation of the binary with respect to the observer. Material at low $\phi$ is ejected earlier and moving faster. We can approximate the bounds on the velocity $v(\phi)$ as $v_{\min }=\max \left[-\frac{4 \phi}{\pi}\langle v\rangle, 0\right]$, $v_{\max }=\min \left[2\left(1-\frac{2 \phi}{\pi}\right)\langle v\rangle, 2\langle v\rangle\right]$. The ripples observed in velocity space are most likely of numerical origin.

observed at an azimuthal angle $\phi$ is

$$
\begin{aligned}
& v_{\min }=\max \left[-\frac{4 \phi}{\pi}\langle v\rangle, 0\right], \\
& v_{\max }=\min \left[2\left(1-\frac{2 \phi}{\pi}\right)\langle v\rangle, 2\langle v\rangle\right],
\end{aligned}
$$

with $\phi$ in the range $[-\pi / 2, \pi / 2]$ covering the regions in which a significant amount of ejecta is present. If the velocity distribution significantly affects our ability to extract useful information about the merging objects from the observation of kilonovae, a more accurate model will certainly have to be designed based on a larger number of simulations (including some ejecting a small amount of material in a larger arc $\delta \phi \sim 2 \pi$, as observed in [27]).

\subsection{Post-merger remnant}

Closer to the black hole, the disrupted neutron star forms a precessing tidal tail, which eventually leads to the formation of a disk over a timescale of $(5-10)$ ms. Rapid accretion onto to black hole stops $\sim 2-3 \mathrm{~ms}$ after merger (we define the merger time as the time at which half the neutron star mass has been accreted onto the black hole). At that time, the accretion rate and average temperature reach clear minima. As this is a convenient feature of all simulations which immediately follows the merger, we choose that time to measure many of the quantities listed in Table 2 (mass and spin of the black hole, baryon mass remaining outside of the black hole). The decrease in accretion rate and temperature stops as the tidal 


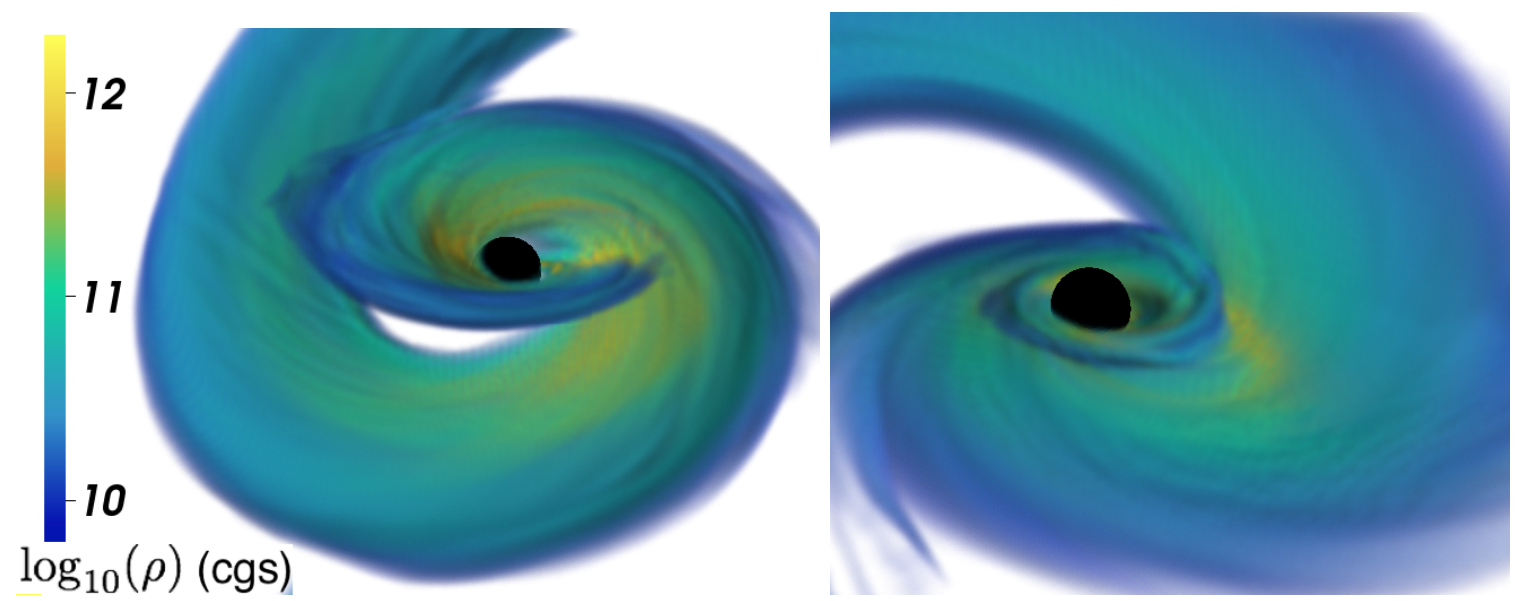

Figure 6. Baryon density at the beginning of disk circularization (2.5 ms after merger) (Left), and $3 \mathrm{~ms}$ later (Right), for model M5-S7-I60. The formation of a wide, hot disk is slow compared to the dynamical timescale of the inner disk: $5.5 \mathrm{~ms}$ after merger, the peak of the surface density of the fluid is still at $r_{\text {peak }} \sim 30 \mathrm{~km}$, while the horizon of the black hole has a radius $r_{\mathrm{BH}} \sim 27 \mathrm{~km}$. The system remains closer to an accreting tidal tail than a circularized disk.

tail self-intersects and shocks (see left panel of Fig. 6, for model M5-S7-I60). This selfinteraction of the tail material begins a slow circularization of the tidal tail into an accretion disk. The right panel of Fig. 6 shows model M5-S7-I60 $5.5 \mathrm{~ms}$ after merger. At that time, the disk remains very far from axisymmetry, and the surface density still peaks right on the horizon of the black hole.

To observe the formation of an accretion disk and its early evolution, we continue the evolution of model M5-S7-I60 up to $17 \mathrm{~ms}$ after merger. The true accretion disk really forms $\sim 10 \mathrm{~ms}$ after merger, with a peak of the mass distribution at a radius $r_{\text {disk }} \sim(50-60) \mathrm{km}$, a temperature $T \sim 3-6 \mathrm{MeV}$, and an electron fraction $Y_{e} \sim 0.1-0.2$. The disk properties are thus very similar to those of non-precessing systems evolved with a different hot, nucleartheory based equation of state [51]. During disk formation, the accretion rate on the black hole remains very high, with $\dot{M}_{\mathrm{BH}} \sim 5 M_{\odot} \mathrm{S}^{-1}$ on average. The inner regions of the disk rapidly align with the equatorial plane of the black hole, as already observed for simpler equations of state [40]. While the tidal tail is initially at a $20^{\circ}-25^{\circ}$ inclination with respect to the equatorial plane of the back hole, that misalignment drops to $\sim 10^{\circ}$ only $10 \mathrm{~ms}$ after merger. This change is not due to accretion (the black hole only accretes $\sim 0.03 M_{\odot}$ during this period), and thus has to be due to a combination of hydrodynamical shocks and/or non-linear damping of the inclination due to the interaction between neighboring regions of the differentially precessing disk (see e.g. [70]).

The more violent disk formation phase is followed by a slower secular evolution of the remnant. For the entire duration of the simulation, the accretion rate onto the black hole remains high, at $\dot{M}_{\mathrm{BH}} \sim 5 M_{\odot} \mathrm{S}^{-1}$. A large fraction of the matter outside of the black hole remains in the bound tidal tail. The accretion disk, which we arbitrarily define as the matter within $\sim 150 \mathrm{~km}$ of the black hole (a convenient choice because the cumulative 

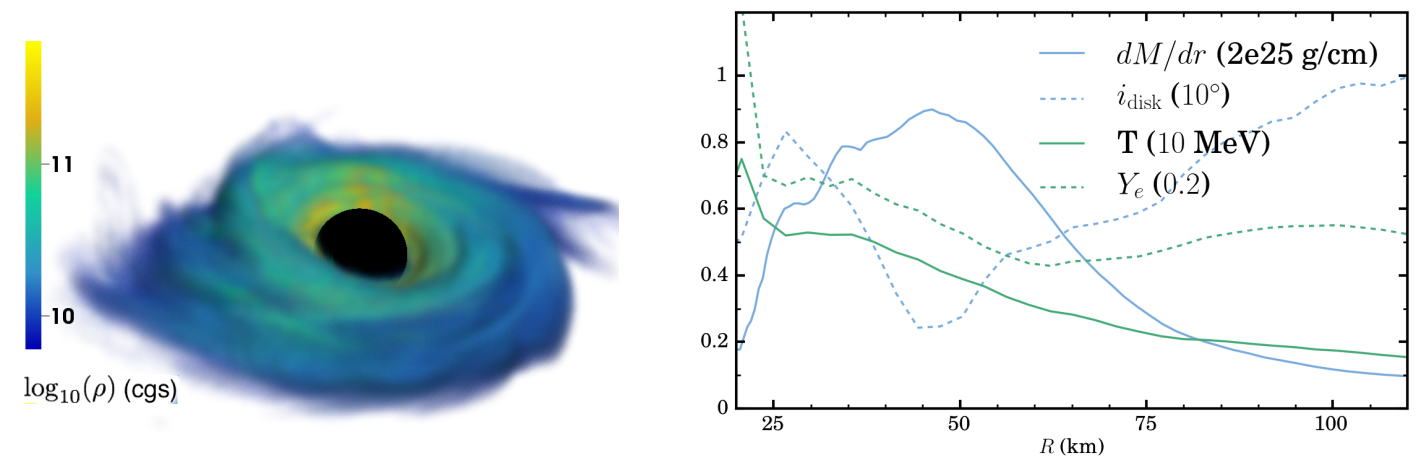

Figure 7. Left: Baryon density $17 \mathrm{~ms}$ after merger for model M5-S7-I60. Right: Onedimensional profile of the linear distribution of the baryon mass $(d M / d r)$, temperature $(T)$, electron fraction $\left(Y_{e}\right)$, and inclination of the disk angular momentum with respect to the black hole spin $\left(i_{\mathrm{BH}}\right)$, at the same time. Number in parenthesis indicate the scale used for each quantity.

mass distribution is nearly flat for $100 \mathrm{~km}<r<300 \mathrm{~km}$ ), has a mass decreasing from $M_{\text {disk }}=0.06 M_{\odot}$ to $M_{\text {disk }}=0.045 M_{\odot}$ between $10 \mathrm{~ms}$ and $17 \mathrm{~ms}$ after the merger. This leaves a bound tidal tail of mass $M_{\text {bound }}=0.027 M_{\odot}$, as well as an ejected mass of $M_{\mathrm{ej}}=0.014 M_{\odot}$ at the end of the simulation. The observed accretion rate can obviously not be sustained for more than another $\sim 10 \mathrm{~ms}$. The fallback rate is thus expected to quickly become an important driver of the evolution of the disk.

We note that with such disk masses, the mass ejected through disk outflows is expected to be only a factor of a few smaller than the mass of the dynamical ejecta, with large uncertainties in both the mass and properties of the outflows. This should be the case for all of the configurations studied here, and indicates that those disk outflows could contribute to the production of a radioactively powered transient.

The temperature of the disk remains constant over the duration of the simulation, despite neutrino luminosities of $L_{\nu} \sim(1.7-3.8) \times 10^{53} \mathrm{erg} / \mathrm{s}$, and the fact that we do not include magnetic fields and thus do not benefit from heating due to magnetic turbulence. In our simulations, the heating is entirely driven by hydrodynamical shocks during the circularization of the material falling back onto the disk. Similarly, after a phase of rapid protonization of the fluid during disk formation, the electron fraction of the disk remains nearly constant. While the disk continuously emits more electron antineutrinos than electron neutrinos, that effect is mostly compensated by the accretion of high- $Y_{e}$ material by the black hole, and the fallback of low $-Y_{e}$ material from the tidal tail. The relatively constant properties of the postmerger remnant indicate that snapshots of our simulation anywhere in the range $(10-17) \mathrm{ms}$ after merger probably provide reasonable initial conditions for long-term evolutions of these disks, during which magnetically-driven turbulence and neutrino transport will play a more important role.

A more detailed view of the final disk is provided in Fig. 7 . We see that the disk is very narrow, with most of the matter within $25 \mathrm{~km}<r<75 \mathrm{~km}$. There also remains a 
mild variance of $\sim 4^{\circ}$ in the orientation of the disk. Otherwise, the disk has settled into a configuration similar to what is observed in other NSBH mergers, with a narrow annulus of nearly axisymmetric material, an inverted temperature gradient (with the inner edge hotter than the outer edge), and a disk height $H \sim 0.2 r$.

Finally, we monitor the evolution of the disk inclination over the entire evolution. We note that the alignment of the angular momentum of the disk with the spin of the black hole continues for the entire duration of the evolution, despite significant accretion of misaligned fallback material. The misalignment angle between the angular momentum of the disk (within a narrow annulus around its density peak) and the spin of the black hole continuously drops from $10^{\circ}$ about $10 \mathrm{~ms}$ after merger to about $3^{\circ}$ at the end of the simulation. There is no noticeable global precession of the disk during that period. The alignment of the disk occurs much faster than in our previous simulations of precessing systems [36], presumably due to the larger black hole mass, larger black hole spin, and smaller disk mass in the simulations presented here.

\section{Comparison with existing fitting formulae}

In recent years, analytical formulae fitted to numerical simulations have been proposed to predict the baryon mass remaining outside of the black hole after NSBH mergers [24, 25], the mass and velocity of the ejected material [41], and the final mass and spin of the black hole [64]. In Table 2, we provide as references the predictions of numerical fits to the remnant baryon mass from [25] (extended to misaligned black hole spins as suggested in [71]), the ejected mass from [41], and the final black hole properties from [64]. We see good qualitative agreements between the analytical predictions and our numerical results. For the low mass systems, the larger remnant masses observed in the simulations are simply due to difference in the time at which the remnant mass is measured (e.g. model M5-S7-I60 has a remnant mass outside of the black hole of $0.12 M_{\odot}$ if we measure it $10 \mathrm{~ms}$ after merger, as for the fitting formula [25]). For higher mass systems with high spins, the fitting formula somewhat underestimates the remnant mass. This is a common issue when the remnant mass is large (see e.g. [72]). Yet the results remain qualitatively correct, and in particular both the simulations and the fit predict that model M7-S7-I60 does not lead to the disruption of the neutron star, while all other models do. The fit to the final mass and spin of the black hole [64] generally performs as well or better than expected. As that fit mostly relies on the knowledge of the baryon mass remaining outside of the black hole [25] and on conservation of energy and angular momentum, the absolute error in the final mass is comparable to the error in the remnant baryon mass. The spins are very accurately predicted for 3 out of the 4 models, while we observe a slightly larger than expected error (0.03) for the final spin of model M7-S9-I20.

The ejected mass is also accurately predicted by the existing fitting formula, at least within the scatter of $\sim 0.01 M_{\odot}$ also observed in the simulations used to calibrate the model [41]. The average velocity shows more significant differences. According to the fitting formula, we would expect an average (root-mean-square) velocity of $\left\langle v_{\mathrm{ej}, \mathrm{rms}}\right\rangle=$ $(0.245,0.267) c$ for black hole masses of $M_{\mathrm{BH}}=(5,7) M_{\odot}$, with $\left\langle v_{\mathrm{ej}, \mathrm{rms}}\right\rangle=\sqrt{2 E_{\mathrm{kin}} / M_{\mathrm{ej}}}$, 
and $E_{\text {kin }}$ the kinetic energy of the ejecta. With the same definition, we have $\left\langle v_{\text {ej,rms }}\right\rangle=$ $(0.175,0.22) c$ in our simulations. The difference between $\left\langle v_{\mathrm{ej}, \mathrm{rms}}\right\rangle$ and the values of $\langle v\rangle$ listed in Table 2 is due to the change of definition of the average velocity. $\left\langle v_{\mathrm{ej}, \mathrm{rms}}\right\rangle$ is the more relevant definition when considering the kinetic energy in the ejecta, which determines e.g. the amount of energy of the radio transients powered by interactions of the ejecta with the interstellar medium [66], while $\left\langle v_{\mathrm{ej}}\right\rangle$ determines the expansion velocity of the ejecta, which has important effects on the duration and luminosity of kilonovae [32, 33]. Even accounting for this difference in the definition of the average energy, however, we are still left with velocities $(20-40) \%$ lower than predicted by the fitting formula [41].

There is however an important difference between the equations of state considered in the simulations used to calibrate the fitting formula [40], and the equation of state used in our simulations. The first are composition independent, and fitted to the equation of cold matter in nuclear statistical equilibrium (NSE) and beta-equilibrium. The second is composition dependent and only assumes NSE. As the ejecta in NS-BH mergers is cold, the ejected material in our simulations expands at (nearly) constant composition. On the other hand, for the piecewise polytropic equations of state used in [40], the ejected material expands in betaequilibrium. Looking at the internal energy of cold, low density matter in the DD2 equation of state, we find that the difference in internal energy between matter at $Y_{e} \sim 0.05$ (as in our ejecta) and matter at $Y_{e} \sim 0.5$ (the effective composition of the piecewise polytropic equations of state at low-density) is $\Delta \epsilon=7.6 \mathrm{MeV} /$ nuc. This is simply due to the fact that cold matter at $Y_{e} \sim 0.05$ is assumed to be formed of mostly neutrons, with $\sim 10 \%$ of low-mass seed nuclei, while matter at $Y_{e} \sim 0.5$ is assumed to be mostly formed of ${ }^{56} \mathrm{Ni}$. Neither of these assumptions is correct: the ejecta in fact drops out of NSE at temperatures $T \sim 0.5 \mathrm{MeV}$, and its composition is then determined by the result of r-process nucleosynthesis.

These different assumptions can account for a large part of the disagreement in the predicted velocity of the ejecta. If all of the energy which would be released by transforming our neutron rich ejecta into ${ }^{56} \mathrm{Ni}$ was converted into kinetic energy, we would get $\left\langle v_{\mathrm{ej}, \mathrm{rms}}\right\rangle=$ $(0.22,0.24) c$, which brings us within the $\sim 10 \%$ relative error expected for the fitting formula.

The correct answer lies somewhere in between the two results. Nucleosynthesis in the ejecta is expected to release $\sim 6 \mathrm{MeV} /$ nuc. How much of that energy is used to accelerate the ejecta depends on the efficiency with which the products of nuclear reactions during r-process nucleosynthesis are thermalized. Thermalization in the ejecta has been studied in some detail late in the evolution of the system (on the timescale for kilonova emission) [33], but not in the first second when most of the r-process heating is occurring. We will here follow [73] and make the rough estimate that about half of the energy released is lost to neutrinos. This is a good approximation if most of the energy is released in beta-decays producing relativistic electrons and neutrinos. With this assumption, we can estimate that the ejecta will have a kinetic energy $\sim 4.6 \mathrm{MeV} /$ nuc lower than predicted using piecewise polytropic equations of state. We can thus derive a new prediction for the average velocity of the ejecta, based on the existing fit [41], by removing $\sim 4.6 \mathrm{MeV} /$ nuc from the estimated kinetic energy:

$$
\left\langle v_{\mathrm{ej}, \mathrm{rms}}\right\rangle=0.0166 * Q+0.1657,
$$




$$
\left\langle v_{\mathrm{ej}}\right\rangle \quad=0.0149 * Q+0.1493 .
$$

For the second formula, we assumed a ratio of $\sim 1.11$ between the root-mean-square and linear average of the velocity, as observed in our simulations. Once differences in the composition of the fluid at low density are taken into account, this estimate is consistent with our numerical simulations and with pre-existing results. We note that while differences in the average velocity of $\delta\langle v\rangle \sim(0.02-0.05) c$ may seem fairly trivial, the properties of kilonovae are very strongly affected by changes in the ejecta velocity [32, 33]. Any attempt to extract information about the ejected mass from an observed kilonova could be significantly biased

if velocities are inaccurately estimated. They may also be affected by the spatial distributions of velocities, which has been idealized in the only existing ejecta model [41].

\section{Conclusions}

We perform the first general relativistic simulations of precessing neutron star-black hole mergers in which the neutron star matter is modeled using a hot, temperature-dependent, nuclear-theory based equation of state. We cover a range of black hole masses expected from the observation of galactic X-ray binaries, and black hole spins chosen to allow for tidal disruption of the neutron star in most simulations. We focus on the dynamical ejection of material by the merger, and the formation of an accretion disk, but do not follow the longterm evolution of the remnant disk. Longer evolutions of the disk would require the inclusion of magnetic effects, and a better treatment of the neutrinos than the simple leakage scheme used in this work.

We find that the disruption (or non-disruption) of the neutron star, the amount of matter ejected by the merger, and the properties of the post-merger remnant (disk mass, black hole mass and spin) are consistent with existing fitting formulae, even those which are extrapolated from the results of non-precessing merger simulations [25, 64]. The ejected mass is also consistent with predictions derived from precessing merger simulations using a simpler equation of state [40, 41]. The velocity of the ejecta, on the other hand, differ significantly from those predictions. We show that this disagreement can be traced to the assumptions made by the different equations of state at low density and temperature, which affect the evolution of the neutron-rich, cold dynamical ejecta. We argue that none of the equations of state used so far properly capture the evolution of that ejecta, but that simple corrections to the velocities extracted from numerical simulations can easily be applied to improve our estimates of the asymptotic ejecta velocity, and reconcile the results of simulations using different equations of state.

The geometry of the disrupting neutron star also appears to differ between compositiondependent equations of state and piecewise-polytropic [40] or polytropic [36, 26] equations of state. The tidally disrupted star forms a narrower tidal tail, thus making the merger significantly harder to resolve numerically (see also [51] for the same effect in non-precessing systems). To partially offset this problem, we developed an improved method to adaptively construct our numerical grid, and only cover with the grid regions in which matter is currently present. This new method improves our ability to resolve these mergers, even though 
numerical errors remain a significant issue in one simulation of a marginally disrupting neutron star around a rapidly spinning black hole (model M7-S9-I60).

We also show that all of the dynamical ejecta produced in these mergers is very neutronrich, and will undergo strong r-process nucleosynthesis. The velocity distribution of the ejecta appears very similar in all of our simulations, with a broad distribution covering the range $0<v<2\langle v\rangle$, and $\langle v\rangle$ the average velocity of the ejecta. We also provide an approximate model for the angular variation in the velocity distribution of the ejecta, which could be helpful in constructing improved lightcurve models for kilonovae. From the post-merger disk mass and ejected mass, we can infer that disk outflows ejecting material from the postmerger remnant over longer timescales [31, 30] are likely to be subdominant when compared to the dynamical ejecta, but not negligible (maybe $\sim 2-3$ times less massive, with large uncertainties on the mass and properties of the disk outflows). As the dynamical ejecta does not obscure the polar regions of the remnant, if less neutron-rich polar disk outflows are generated they could power a significant optical component to the kilonova signal.

Finally, our results indicate that the remnant accretion disk rapidly aligns with the equatorial plane of the spinning black hole remnant, as in simulations using piecewise polytropic equations of state [40]. For $M_{\mathrm{BH}}=5 M_{\odot}$, the bound material forms a narrow, hot, and compact accretion torus, with most of the mass at radii $25 \mathrm{~km}<r<75 \mathrm{~km}$. Continuous fallback onto the disk causes shocks in the post-merger remnant which heat the disk and drive strong accretion onto the black hole $\left(\dot{M} \sim 5 M_{\odot} / s\right)$, and sustained neutrino emission $\left(L_{\nu}>10^{53} \mathrm{erg} / \mathrm{s}\right)$. This provides ideal initial conditions for the production of hotter disk outflows and/or short gamma-ray bursts. The long-term evolution of this remnant cannot however be reliably performed with the limited physics included in these simulations.

\section{Acknowledgments}

The authors thank Jennifer Barnes, Rodrigo Fernandez, Brian Metzger, Eliot Quataert, Sasha Tchekhovskoy, and the members of the SxS collaboration for helpful discussions over the course of this project. We also thank Francesco Pannarale for providing information about the predicted properties of the final black holes, listed in Table 2. Support for this work was provided by NASA through Einstein Postdoctoral Fellowship grant numbered PF4-150122 (F.F.) awarded by the Chandra X-ray Center, which is operated by the Smithsonian Astrophysical Observatory for NASA under contract NAS8-03060. D.D. gratefully acknowledges support from the UC Berkeley-Rose Hills Foundation Summer Undergraduate Research Fellowship. D.K. is supported in part by a Department of Energy Office of Nuclear Physics Early Career Award, and by the Director, Office of Energy Research, Office of High Energy and Nuclear Physics, Divisions of Nuclear Physics, of the U.S. Department of Energy under Contract No. DE-AC02-05CH11231. H.P. gratefully acknowledges support from the NSERC Canada. M.D. acknowledges support through NSF Grant PHY-1402916. L.K. acknowledges support from NSF grants PHY-1306125 and AST1333129 at Cornell, while the authors at Caltech acknowledge support from NSF Grants PHY1404569, AST-1333520, NSF-1440083, and NSF CAREER Award PHY-1151197. Authors 
at both Cornell and Caltech also thank the Sherman Fairchild Foundation for their support. Computations were performed on the supercomputer Briarée from the Université de Montréal, managed by Calcul Québec and Compute Canada. The operation of these supercomputers is funded by the Canada Foundation for Innovation (CFI), NanoQuébec, RMGA and the Fonds de recherche du Québec - Nature et Technologie (FRQ-NT). Computations were also performed on the Zwicky cluster at Caltech, supported by the Sherman Fairchild Foundation and by NSF award PHY-0960291.

[1] Aasi J et al. (LIGO Scientific Collaboration) 2015 Class. Quantum Grav. 32074001 (Preprint 1411. 4547)

[2] Acernese F et al. (Virgo Collaboration) 2015 Class. Quantum Grav. 32024001 (Preprint 1408.3978)

[3] Aso Y, Michimura Y, Somiya K, Ando M, Miyakawa O, Sekiguchi T, Tatsumi D and Yamamoto H (The KAGRA Collaboration) 2013 Phys. Rev. D 88(4) 043007 (Preprint 1306.6747) URL http: //link.aps.org/doi/10.1103/PhysRevD.88.043007

[4] Abbott B P et al. (LIGO Scientific Collaboration, Virgo Collaboration) 2016 Phys. Rev. Lett. 116(6) 061102 (Preprint 1602.03837) URL http://link.aps.org/doi/10.1103/PhysRevLett.116. 061102

[5] Abbott B P et al. (Virgo, LIGO Scientific) 2016 Phys. Rev. Lett. 116241103 (Preprint 1606.04855 )

[6] The LIGO Scientific Collaboration and the Virgo Collaboration 2016 ArXiv e-prints (Preprint 1606. 04856)

[7] Abadie J et al. (LIGO Scientific Collaboration) 2010 Class. Quantum Grav. 27173001 (Preprint 1003. 2480)

[8] Mochkovitch R, Hernanz M, Isern J and Martin X 1993 Nature 361236

[9] Lee W H and Kluźniak W 1998 Black hole-neutron star coalescence as a source of gamma-ray bursts Gamma-Ray Bursts, 4th Hunstville Symposium (American Institute of Physics Conference Series vol 428) ed C A Meegan, R D Preece, \& T M Koshut p 798

[10] Janka H T, Eberl T, Ruffert M and Fryer C L 1999 Astrophys. J. 527 L39-L42 (Preprint astro-ph/ 9908290)

[11] Fong W and Berger E 2013 Astrophys. J. 77618 (Preprint 1307.0819)

[12] Lattimer J M and Schramm D N 1976 Astrophys. J. 210 549-567

[13] Li L X and Paczynski B 1998 Astrophys. J. 507 L59 (Preprint astro-ph/9807272)

[14] Roberts L F, Kasen D, Lee W H and Ramirez-Ruiz E 2011 Astrophys. J. Lett. 736 L21 (Preprint 1104.5504

[15] Kasen D, Badnell N R and Barnes J 2013 Astrophys. J. 77425 (Preprint 1303.5788)

[16] Tanaka M and Hotokezaka K 2013 Astrophys. J. 775113 (Preprint 1306.3742 )

[17] Korobkin O, Rosswog S, Arcones A and Winteler C 2012 Mon. Not. Roy. Astr. Soc. 4261940 (Preprint 1206.2379)

[18] Wanajo S, Sekiguchi Y, Nishimura N, Kiuchi K, Kyutoku K and Shibata M 2014 Astrophys.J.Lett. 789 L39 (Preprint 1402.7317)

[19] Read J S, Markakis C, Shibata M, Uryū K, Creighton J D E and Friedman J L 2009 Phys. Rev. D 79124033 (Preprint 0901.3258)

[20] Del Pozzo W, Li T G F, Agathos M, Van Den Broeck C and Vitale S 2013 Phys. Rev. Lett. 111071101 (Preprint 1307.8338 )

[21] Lackey B D and Wade L 2015 Phys. Rev. D 91043002 (Preprint 1410.8866 )

[22] Clark J A, Bauswein A, Stergioulas N and Shoemaker D 2016 Class. Quant. Grav. 33085003 (Preprint arXiv:1509.08522 [astro-ph.HE])

[23] Takami K, Rezzolla L and Baiotti L 2015 Phys. Rev. D 91064001 (Preprint 1412.3240 )

[24] Pannarale F, Tonita A and Rezzolla L 2011 Astrophys.J. 72795 (Preprint 1007.4160)

[25] Foucart F 2012 Phys. Rev. D 86124007 (Preprint 1207.6304 )

[26] Foucart F, Deaton M B, Duez M D, Kidder L E, MacDonald I, Ott C D, Pfeiffer H P, Scheel M A, Szilagyi B and Teukolsky S A 2013 Phys. Rev. D 87084006 (Preprint 1212.4810) 
[27] Kyutoku K, Ioka K, Okawa H, Shibata M and Taniguchi K 2015 Phys.Rev.D 92044028 (Preprint 1502.05402 )

[28] Kiuchi K, Sekiguchi Y, Kyutoku K, Shibata M, Taniguchi K and Wada T 2015 Phys. Rev. D 92064034 (Preprint 1506.06811 )

[29] Dessart L, Ott C D, Burrows A, Rosswog S and Livne E 2009 Astrophys. J. 690 1681-1705 (Preprint 0806.4380 )

[30] Just O, Bauswein A, Ardevol Pulpillo R, Goriely S and Janka H T 2015 Mon. Not. Roy. Astr. Soc. 448 541-567 (Preprint 1406.2687 )

[31] Fernández R and Metzger B D 2013 Mon. Not. Roy. Astr. Soc. $435502-517$ (Preprint 1304.6720)

[32] Barnes J and Kasen D 2013 Astrophys. J. 77518 (Preprint 1303.5787)

[33] Barnes J, Kasen D, Wu M R and Mart'inez-Pinedo G 2016 ArXiv e-prints (Preprint 1605.07218)

[34] Lippuner J and Roberts L F 2015 ApJ 81582 (Preprint 1508.03133)

[35] East W E, Paschalidis V and Pretorius F 2015 ApJ Letters 807 L3 (Preprint 1503.07171)

[36] Foucart F, Duez M D, Kidder L E and Teukolsky S A 2011 Phys. Rev. D 83024005 (Preprint arXiv: 1007.4203 [astro-ph.HE])

[37] Kawaguchi K, Kyutoku K, Nakano H, Okawa H, Shibata M and Taniguchi K 2015 Phys. Rev. D 92(2) 024014 URL http://link.aps.org/doi/10.1103/PhysRevD.92.024014

[38] Rantsiou E, Kobayashi S, Laguna P and Rasio F 2008 Astrophys. J. 680 1326-1349 (Preprint arXiv: astro-ph/0703599)

[39] Hempel M, Fischer T, Schaffner-Bielich J and Liebendörfer M 2012 Astrophys.J. 74870 (Preprint 1108.0848 )

[40] Kawaguchi K, Kyutoku K, Nakano H, Okawa H, Shibata M and Taniguchi K 2015 Phys. Rev. D 92024014 (Preprint 1506.05473 )

[41] Kawaguchi K, Kyutoku K, Shibata M and Tanaka M 2016 Astrophys. J. 82552 (Preprint 1601.07711 )

[42] Paschalidis V, Ruiz M and Shapiro S L 2015 Astrophys. J. 806 L14 (Preprint 1410 . 7392)

[43] Foucart F, OConnor E, Roberts L, Duez M D, Haas R, Kidder L E, Ott C D, Pfeiffer H P, Scheel M A and Szilagyi B 2015 Phys. Rev. D 91124021 (Preprint 1502.04146)

[44] Ruffert M, Janka H T and Schaefer G 1996 Astron. Astrophys. 311 532-566 (Preprint astro-ph/ 9509006)

[45] Rosswog S and Liebendörfer M 2003 Mon. Not. Roy. Astr. Soc. 342673 (Preprint ast ro-ph/ 0302301 )

[46] O'Connor E and Ott C D 2010 Class. Quantum Grav. 27114103 (Preprint 0912.2393)

[47] Foucart F, Haas R, Duez M D, O'Connor E, Ott C D, Roberts L, Kidder L E, Lippuner J, Pfeiffer H P and Scheel M A 2016 Phys.Rev.D. 93044019 (Preprint 1510.06398 )

[48] Sekiguchi Y, Kiuchi K, Kyutoku K and Shibata M 2015 Phys. Rev. D 91064059 (Preprint 1502.06660 )

[49] Sekiguchi Y, Kiuchi K, Kyutoku K, Shibata M and Taniguchi K 2016 Phys. Rev. D93 124046 (Preprint 1603.01918)

[50] Deaton M B, Duez M D, Foucart F, O'Connor E, Ott C D, Kidder L E, Muhlberger C D, Scheel M A and Szilagyi B 2013 Astrophys. J. 77647 (Preprint 1304.3384 )

[51] Foucart F, Deaton M B, Duez M D, O'Connor E, Ott C D, Haas R, Kidder L E, Pfeiffer H P, Scheel M A and Szilagyi B 2014 Phys. Rev. D 90024026 (Preprint 1405.1121 )

[52] Steiner A W, Lattimer J M and Brown E F 2013 ApJ Letters 765 L5 (Preprint 1205.6871)

[53] Özel F, Psaltis D, Narayan R and McClintock J E 2010 Atrophys.J. 725 1918-1927 (Preprint 1006.2834 )

[54] Kreidberg L, Bailyn C D, Farr W M and Kalogera V 2012 Astroph.J. 75736 (Preprint 1205.1805)

[55] Pfeiffer H P, Kidder L E, Scheel M A and Teukolsky S A 2003 Comput. Phys. Commun. 152 253-273 (Preprint gr-qc/0202096)

[56] Foucart F, Kidder L E, Pfeiffer H P and Teukolsky S A 2008 Phys. Rev. D 77124051 (Preprint arXiv: 0804.3787 )

[57] Gourgoulhon E, Grandclément P, Taniguchi K, Marck J A and Bonazzola S 2001 Phys. Rev. D 63064029 ISSN 0556-2821 URL http://link.aps.org/doi/10.1103/PhysRevD.63.064029

[58] Taniguchi K, Baumgarte T W, Faber J A and Shapiro S L 2006 Phys. Rev. D 74041502

[59] http://www.black-holes.org/SpEC.html 
[60] Lindblom L, Scheel M A, Kidder L E, Owen R and Rinne O 2006 Class. Quantum Grav. 23 S447 (Preprint gr-qc/0512093v3)

[61] Szilágyi B 2014 Int. J. Mod. Phys. D 231430014 (Preprint 1405 . 3693)

[62] Lovelace G, Owen R, Pfeiffer H P and Chu T 2008 Phys. Rev. D 78084017

[63] Duez M D, Foucart F, Kidder L E, Pfeiffer H P, Scheel M A and Teukolsky S A 2008 Phys. Rev. D 78 104015 (Preprint 0809.0002 )

[64] Pannarale F 2014 Phys. Rev. D 89044045 (Preprint 1311.5931 )

[65] Roberts L F, Lippuner J, Duez M D, Faber J A, Foucart F, Lombardi Jr J C, Ning S, Ott C D and Ponce M 2016 arXiv:1601.07942 (Preprint 1601.07942 )

[66] Nakar E and Piran T 2011 Nature 478 82-84 (Preprint 1102.1020 )

[67] Perego A, Rosswog S, Cabezón R M, Korobkin O, Käppeli R, Arcones A and Liebendörfer M 2014 Mon. Not. Roy. Astr. Soc. 443 3134-3156 (Preprint 1405.6730 )

[68] Fernández R, Kasen D, Metzger B D and Quataert E 2015 Mon. Not. Roy. Astr. Soc. $446750-758$ (Preprint 1409.4426 )

[69] Fernández R, Quataert E, Schwab J, Kasen D and Rosswog S 2015 Mon. Not. Roy. Astron. Soc. 449390 402 (Preprint 1412.5588 )

[70] Sorathia K A, Krolik J H and Hawley J F 2013 ApJ 768133 (Preprint 1303.5465 )

[71] Stone N, Loeb A and Berger E 2013 Phys. Rev. D 87084053 (Preprint 1209.4097)

[72] Lovelace G, Duez M D, Foucart F, Kidder L E, Pfeiffer H P, Scheel M A and Szilágyi B 2013 Class. Quantum Grav. 30135004 (Preprint 1302 . 6297)

[73] Metzger B D, Arcones A, Quataert E and Martínez-Pinedo G 2010 Mon. Not. Roy. Astr. Soc. 402 27712777 (Preprint 0908.0530 )

[74] Hemberger D A, Scheel M A, Kidder L E, Szilágyi B, Lovelace G, Taylor N W and Teukolsky S A 2013 Class. Quantum Grav. 30115001 (Preprint 1211.6079) URL http: / / stacks.iop.org/ $0264-9381 / 30 / i=11 / a=115001$

\section{Appendix A. Adaptive grid structure}

An important difficulty when simulating NSBH mergers with hot nuclear-theory based equations of state is that, when the neutron star disrupts close to the ISCO, the fluid forms a very narrow, rapidly moving tidal tail [51]. The difference in length scale between the width of the tail $\sim 5 \mathrm{~km}$, the radius of the forming accretion disk $\sim 50 \mathrm{~km}$, and the distance to which we have to follow the ejected material to reliably extract its properties $\sim 1000 \mathrm{~km}$ makes it computationally impossible to study these mergers without the use of mesh refinement techniques. Previously, we used a fixed mesh refinement method, with a grid composed of nested boxes of increasing resolution centered on the black hole (with a factor of two change in resolution between each level of refinement). During the disruption of the neutron star, this is very suboptimal, as the narrow tidal tail has a very low filling factor in the cubic grid, $(10-20) \%$, before formation of an accretion disk. This leads to wasted numerical resources in a phase of the merger during which a high numerical resolution is required to avoid numerical shocks in the contracting tidal tail.

To solve this issue, we now let SpEC automatically turn small sections of the computational domain on and off, as matter moves through the grid. Because SpEC evolves the spacetime metric on a separate grid, there is no need for the finite volume grid to extend to regions where no matter is present. In practice, during the disruption of the neutron star, 

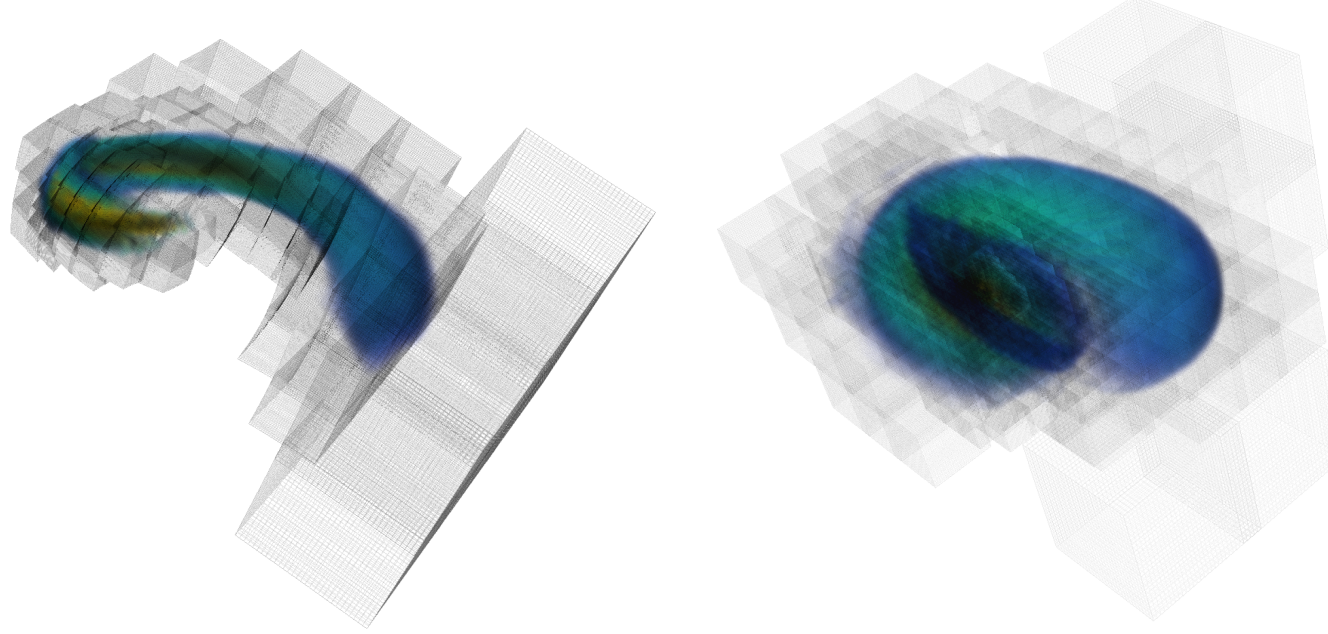

Figure A1. Grid structure and baryon density during the disruption of the neutron star (left), and after disk formation (right). Both images are snapshots of model M5-S7-I60, with the color scale showing the logarithm of the baryon density wherever $\rho \gtrsim 10^{10} \mathrm{~g} / \mathrm{cm}^{3}$. The advantage of an adaptive grid is clearly visible during disruption. After merger, the technique remains advantageous in following the tidal tail, while the inner regions are more densely filled with disk material.

we use 7 levels of refinement, each with $200^{3}$ points $f$ Each level is subdivided into $8^{3}$ subdomains, which can be individually turned on and off. The central $4^{3}$ subdomains of each level are always turned off, except at the finest level of refinement. (They overlap subdomains with a finer mesh.) A subdomain is turned on when, anywhere within 6 grid spacings of its outer boundary, the fluid density satisfies

$$
\rho>\rho_{F M R}=3 \times 10^{10}\left[0.001+\left(\frac{2 r_{\mathrm{exc}}}{r+r_{\mathrm{exc}}}\right)^{2}\right] \mathrm{g} \mathrm{cm}^{-3},
$$

with $r_{\text {exc }}$ the excision radius and $r$ the coordinate distance to the black hole center in grid coordinates. A subdomain is turned off as soon as all grid cells within 6 grid spacings of its boundary satisfy the condition $\rho<\left(\rho_{\mathrm{FMR}} / 2\right)$. We test these conditions every 20 time steps, and modify the domain (including rebalancing the computational load) whenever at least one subdomain needs to be turned on. We note that, while this method allows for more efficient evolutions, it does not match the flexibility of a true Adaptive Mesh Refinement technique. In our algorithm, the structure of the numerical grid is fixed by the user, and the code can only choose whether to ignore a region of spacetime or not. In essence, this is thus still a Fixed Mesh Refinement algorithm, but one which is capable of ignoring the large fraction of the numerical grid in which no matter is present in neutron star-black hole mergers.

Examples of the resulting domain decomposition are shown on Fig. A1. During tidal disruption, our algorithm significantly reduces the cost of the evolution by only using

$\ddagger$ These 7 refinement levels form a purely potential grid - during the simulations, we never need to turn on subdomains in more than 5 levels of refinement at any given time. 
subdomains covering the fluid. After disk formation, we still gain by only turning on subdomains covering the disk and tidal tail, but the filling factor of the matter is now significantly larger $(50 \%-80 \%$ at the finest level of refinement). Once a disk forms, the resolution requirements to accurately evolve the fluid become less stringent. We remove the finest level of refinement around the time at which the mass accretion rate onto the black hole reaches its first local minimum (i.e. before circularization of the disk material begins), and the second finest level once a more axisymmetric disk has been formed.

This adaptive mesh is only used during tidal disruption (or plunge) of the neutron star and for the post-merger evolution of the remnant. Before merger, we use a uniform grid with spacing $\Delta x=220 \mathrm{~m}$ in the coordinates of the finite volume grid. To determine the grid spacing in the laboratory frame, we have to remember that SpEC uses a time-dependent map between the grid coordinates and the laboratory frame. A first map keeps the center of the compact objects fixed in the coordinates of the pseudospectral grid on which we evolve Einstein's equations, and makes sure that the excised region inside the apparent horizon of the black hole is of a shape and size guaranteeing that no information flows from the boundary into the computational domain [74]. A second map relates the coordinates of the finite volume grid to the coordinates of the pseudospectral grid, and keeps the neutron star fluid entirely within the finite volume grid. The grid spacing in the laboratory frame is thus time-dependent. Before merger, the grid spacing will change by $\sim 25 \%$ around the initial value $\Delta x=220 \mathrm{~m}$, with a better resolution in the directions in which tides cause the neutron star to contract, and a lower resolution in the direction in which it expands.

During the tidal disruption of the neutron star and the post-merger evolution, the finite volume grid and pseudospectral grid use the same coordinate system. The adaptive grid described above is used to keep the neutron star fluid on the finite volume grid. We still use a time-dependent map between the grid coordinates and the laboratory frame, to control the location, shape, and size of the apparent horizon of the black hole. We keep the grid spacing of the finest level of refinement in the grid coordinates to $\Delta x \approx 250 \mathrm{~m}$. Because the grid coordinates contracted as the binary inspiralled, this generally corresponds to a finer resolution in the laboratory frame, which will be different for each simulation. We provide the typical value of the finest grid spacing during disruption for simulations at our fiducial resolution in Table 1, and discuss lower resolution simulations and the inferred numerical accuracy of our results in Sec. Appendix B.

\section{Appendix B. Numerical accuracy}

Beyond the fiducial simulations using an adaptive grid described in Appendix A, we evolve each model at one or more lower resolution, using fixed finite volume grids with 2-3 levels of refinement. These lower resolution simulations typically use $\Delta x_{\text {dis }} \approx(250-300) \mathrm{m}$ for the grid spacing $\Delta x_{\text {dis }}$ of the finest refinement level in the laboratory frame, instead of $\Delta x_{\text {dis }} \approx(150-200) \mathrm{m}$ with the adaptive grid (see Table 1$)$. We use these lower resolution simulations to get estimates of our numerical accuracy.

The main source of error in our simulations is the evolution of the fluid close to the 
horizon of the black hole, and thus to the surface of the region inside the black hole which is excised from our computational domain. For fluid elements which plunge into the black hole and are causally disconnected from the rest of the matter, this has no practical effect on the evolution. However, for models in which the tidal disruption of the neutron star occurs very close to the black hole, this is a significant source of error. As for higher mass systems tidal disruption occurs closer to the black hole horizon, numerical errors are typically larger. This is compounded by the fact that the disrupted neutron star forms a narrow, hard-to-resolve tidal tail with a sharp front edge moving at supersonic speeds through the grid. This difficulty in resolving the disruption of neutron stars described by hot, nuclear-theory based equations of state was already noted for aligned-spin binaries [51]. Merger simulations with polytropic and piecewise-polytropic equations of state, on the other end, do not appear to show the creation of such narrow tails, making their disruption significantly easier to simulate.

The worst manifestation of this numerical error is the ejection at high velocity of some material which would otherwise fall into the black hole, due to numerical shocks in the underresolved tidal tail. This effect is clearly visible in our lower resolution simulations for all simulations with $M_{\mathrm{BH}}=7 M_{\odot}$, and is the main motivation to use an adaptive grid and increase the resolution of our simulations (the evolution of the fluid at distances larger than $\sim 1.5 r_{\mathrm{H}}$, with $r_{H}$ the radius of the horizon, is well converged for all of our simulations). This numerical ejecta can be easily distinguished from the physical dynamical ejecta, as it originates in a completely different part of the disrupting neutron star. It clearly converges away at highresolution, except for model M7-S9-I60. Model M7-S9-I60 is the most problematic case because it is a relatively high mass ratio, and a case which is only marginally disrupting. The production of a few percents of a solar mass of unphysical ejecta even at a resolution $\Delta x \approx 100 \mathrm{~m}$ significantly affects measurements of the ejected mass and, potentially, baryon mass outside of the black hole. Accordingly, we do not report post-merger quantities for that model.

For all other disrupting models, the mass of the dynamical ejecta converges to $\lesssim 20 \%$, while the baryon mass outside of the black hole and the other reported properties of the disk and ejecta (average velocities, composition, temperature, inclination) converge to $\lesssim 10 \%$. Finally, for the non-disrupting model M7-S7-I60, we report upper bounds on the ejected mass and baryon mass outside the black hole, obtained from the highest resolution simulation that we performed. 